\title{
Bowel Preparation for Small Bowel Capsule Endoscopy: Is There Still a Role for Polyethylene Glycol?
}

\author{
Paul Collins ${ }^{1}$ Neil Haslam¹ Anthony Morris ${ }^{1}$ Thomas Skouras ${ }^{2}$ Ashley Bond ${ }^{1}$ \\ ${ }^{1}$ Department of Gastroenterology and Hepatology, Royal Liverpool \\ University Hospital, Liverpool, United Kingdom \\ 2Department of Gastroenterology and Hepatology, Warrington \\ Hospital, Warrington, United Kingdom

\begin{abstract}
Address for correspondence Paul Collins, MD, FRCP, Department of Gastroenterology and Hepatology, Royal Liverpool University Hospital, Prescot Street, Liverpool L7 8XP, United Kingdom (e-mail: Paul.collins@rlbuht.nhs.uk).
\end{abstract}

\begin{abstract}
Keywords

- bowel cleansing

- bowel preparation

- small bowel capsule endoscopy

- iron-deficiency anemia
\end{abstract}

Objectives This study aimed to assess the impact of polyethylene glycol (PEG) bowel cleansing on performance characteristics of small bowel capsule endoscopy (SBCE). Materials and Methods Data from consecutive patients undergoing SBCE in the period before and after the introduction of PEG $2 \mathrm{~L}$ bowel cleansing with PEG were collated retrospectively. The indication, diagnostic yield (DY), clinical outcome, small bowel transit time, gastric transit time, and completion rate were recorded for each procedure.

Results Data from 286 patients were analyzed. PEG $2 \mathrm{~L}$ was not superior to 12-hour fasting for DY (66 [53\%] vs. 76 [47\%] patients [ $p=0.348]$ ), or DY for significant findings (findings requiring a further intervention or investigation; 29 [23\%] vs. 52 [32\%] patients $[p=0.090])$. There was a trend toward an increased DY for significant findings in patients undergoing investigation for iron-deficiency anemia (IDA) receiving PEC $2 \mathrm{~L}$ that just failed to meet statistical significance (13 [31\%] and 25 [21\%] patients, respectively $[p=0.06])$. Transit times and completion rates were unaffected by bowel cleansing.

Conclusion Bowel cleansing with PEG $2 \mathrm{~L}$ is not superior to fasting for overall DY in SBCE. PEG $2 \mathrm{~L}$ may confer an advantage for the detection of significant lesions in patient with IDA. Further investigation of optimal modes of bowel preparation is indicated.

\section{Introduction}

In the last two decades, small bowel capsule endoscopy (SBCE) has evolved as an important tool for the noninvasive investigation of the small bowel. Its use complements radiological investigations of the small bowel. It has the advantage of having an increased sensitivity for the detection of subtle mucosal lesions that may be missed by other techniques or for the detection of lesions which are relatively inaccessible to flexible endoscopy. ${ }^{1,2}$

The optimization of factors that improve mucosal visualization is of central importance in enhancing the diagnostic yield (DY) of SBCE. However, the evaluation of the evidence for interventions used to improve DY is hampered by the use of varying definitions of DY and by the use of heterogeneous measures of the quality of small bowel visualization.

Attempts have been made to address the role of purgatives to enhance the performance of SBCE. Three recent meta-analyses of studies investigating the role of purgative agents in SBCE have yielded mixed results for outcomes of DY, mucosal visualization, and procedural completion rates. ${ }^{3-5}$ Two consensus guidelines have recommended the use of bowel preparation prior to SBCE. ${ }^{6,7}$ European guidelines specify the use of 2L of polyethylene glycol (PEG) to enhance views of the small bowel in patients undergoing SBCE. ${ }^{7}$ However, within these guidelines, the authors acknowledged that the 
evidence for the impact of PEG on DY and rates of completion was not conclusive. It was also acknowledged that the optimal timing for the use of bowel cleansing agents had not been established. ${ }^{5,8-10}$ In addition to this, patients using PEG as a bowel purgative experience increased nausea, abdominal discomfort, dizziness, and bloating. ${ }^{11,12}$ This may impact negatively on the acceptability of SBCE as a diagnostic tool to patients. A strong case can be made for reexamining the role of bowel purgatives in SBCE.

In this retrospective review of data from SBCE procedures we have investigated the impact of bowel purgatives in the form of PEG on the performance characteristics of SBCE.

\section{Methods}

Small bowel cleansing using PEG was introduced in November 2015. Data from consecutive patients undergoing SBCE between November 2014 and September 2016 were collated and reviewed retrospectively.

All procedures were performed using the small bowel video capsule PillCam SB3 (Given Imaging, Israel). The PillCam SB3 system has been used at our institution since 2014. Previous iterations of the system have been in use at our hospital since 2003.

Patients at increased risk of capsule retention (known Crohn's disease, suspected Crohn's disease, and prior abdominal surgery or those with significant pain) underwent patency capsule assessment (GivenAGILE Patency System) in the 3 months prior to SBCE assessment. ${ }^{7}$ Patients attended the hospital 28 hours after swallowing the patency capsule. A hand-held radiofrequency detector was used to check if the capsule had been excreted. Patients in whom the patency capsule was detected underwent a low-dose computed tomography (CT) scan to determine whether the capsule had passed into the colon. A satisfactory patency test was one in which the patency capsule had been completely excreted or had passed into the colon at the time of assessment.

Patients were instructed to have a low residue diet for 48 hours before taking PEG. On the day before the test, patients were permitted to consume a light breakfast and clear fluids thereafter. A total of 2 L of PEG (MoviPrep, Norgine) was taken the day before the test ( $1 \mathrm{~L}$ at 2 p.m. and $1 \mathrm{~L}$ at 6 p.m.). Patients had no further oral intake for 10 hours before ingestion of the capsule. Prior to the introduction of PEG bowel cleansing for SBCE examination in November 2015, preparation for the procedure was limited to a 12-hour fasting before ingestion of the capsule.

For all examinations, the capsule was swallowed with approximately $50 \mathrm{~mL}$ of water containing $80 \mathrm{mg}$ of the antifoaming agent, Simethicone. Patients were permitted clear fluids 2 hours after capsule ingestion and allowed to eat and drink after 4 hours. Prokinetic agents were not given.

Capsule studies were read by one of three experienced consultant gastroenterologists (P.C., N.H., and A.M.). Retrospective retrieval and collation of capsule data was undertaken by two clinicians (T.S. and A.B.).
The data collected included patient demographics, the indication for SBCE, procedural findings, and recommendations for further investigation or treatment. In those studies in which inflammatory activity was seen, the inflammatory burden was calculated using the Lewis score (LS). ${ }^{13}$ The LS significant inflammation was defined as a LS of greater than 135. ${ }^{14}$ Transit times and the position of the capsule in the gastrointestinal (GI) tract in the final image of the recording were collated.

Capsule retention was excluded in those patients in whom the capsule failed to leave the small bowel during the recording period. A plain abdominal X-ray was performed more than 2 weeks after the completion of the SBCE in this group to confirm successful passage of the capsule.

Technical information on transit times was collected and correlated with bowel cleansing and age.

Significant findings were defined as those for which a further intervention or investigation (endoscopic, radiologic, or surgical) was required.

\section{Outcome Measures}

The DY for SBCE was compared between the group receiving PEG and the group undergoing a 12-hour fasting. The DY for any findings and for significant findings was also determined.

The detection of inflammatory activity and investigation of anemia constitute the main indications for capsule endoscopy. ${ }^{15}$ The DY for the indication of iron-deficiency anemia (IDA) was compared between the PEG group and the group undergoing a 12-hour fasting. The DY for any inflammatory lesion and for significant inflammation (LS > 135) was calculated for both groups.

Gastric and small bowel transit times, and SBCE completion rates (passage of the capsule into the colon or ileostomy bag) were compared between the group receiving PEG and the group undergoing a 12-hour fasting. The effect of age on SBCE transit times was determined. The effect of age and the total recording time of the procedure on the completion rate was also determined.

Ethical approval for this study was given by the local Clinical Effectiveness Group in line with national guidance for the governance and ethical review of health and social care service evaluation in the United Kingdom. ${ }^{16}$

\section{Statistics}

The normality of data was assessed using the Shapiro-Wilk test statistic. Where data were normally distributed, the mean value (standard deviation [SD]) was used to indicate the central tendency and variability of data. Median (range) was used to express the central tendency and variability of nonnormally distributed data. ${ }^{17}$ Mann-Whitney $U$ and independent samples $t$-tests were used to compare groups where appropriate. Correlation of data was assessed using the Spearman's rank correlation for nonparametric data (two-tailed significance). The Chi-square test was used to determine whether there was a significant relationship between categorical variables. A $p$-value of $<0.05$ was 
considered significant(Software:IBMSPSSStatistics,IBMCorp., Version 24.0.; Armonk, New York, United States).

\section{Results}

\section{Demographic and Clinical Data}

- Table 1 summarizes the demographic and clinical data of patients undergoing SBCE. 286 patients underwent SBCE between November 2014 and September 2016. The mean age was 51.5 years $(\mathrm{SD}=18.6)$. There were 166 female patients (58\%) and 120 male patients (42\%). The indications for SBCE were suspected Crohn's disease in 94 (33\%) patients, IDA in 91 (32\%) patients, obscure overt GI blood loss in 34 (12\%) patients, assessment of Crohn's disease in 23 (8\%) patients, polyposis syndrome surveillance in 13 (5\%) patients, radiological abnormality in $6(2 \%)$ patients, possible polyp on imaging in $5(2 \%)$ patients, and assessment in suspected refractory celiac disease in $3(1 \%)$ patients. Other indications accounted for the remaining 17 (6\%) patients.

A total of 283 (98\%) patients swallowed the capsule. One patient was unable to swallow the capsule and required endoscopic placement of the capsule into the duodenum. The capsule was transferred from the stomach to the duodenum after ingestion of the capsule in two patients in whom the capsule had previously failed to leave the stomach in a prior examination.

Also, 125 (44\%) patients underwent PEG bowel cleansing in preparation for the procedure and 161 (56\%) patients underwent a 12-hour fasting.

Complete small bowel examination was achieved in 247 (87\%) patients. At the end of the recording time, the capsule had passed to the colon in $242(85 \%)$ patients and into an ileostomy bag in 5 (2\%) patients. The capsule was located in the small bowel at the end of the recording period in

Table 1 Demographic and clinical data of patients undergoing SBCE

\begin{tabular}{|c|c|c|c|}
\hline & All patients & 12-hour fasting & PEG \\
\hline Total & 286 & 161 & 125 \\
\hline \multicolumn{4}{|l|}{ Sex } \\
\hline Male & $120(42)$ & $67(42)$ & $53(42)$ \\
\hline Female & $166(58)$ & $94(58)$ & $72(58)$ \\
\hline Age $(y)^{\mathrm{a}}$ & $51.5(18.6)$ & $52.1(18.6)$ & $50.8(18.7)$ \\
\hline \multicolumn{4}{|l|}{$\begin{array}{l}\text { Indication } \\
n(\%)\end{array}$} \\
\hline Assessment of Crohn's disease & $23(8)$ & $9(5.6)$ & $14(11.2)$ \\
\hline Suspected Crohn's disease & $94(32.9)$ & $57(35.4)$ & $37(29.6)$ \\
\hline Radiological abnormality & $6(2.1)$ & $1(0.6)$ & $5(4)$ \\
\hline Iron-deficiency anemia & $91(31.8)$ & $49(30.4)$ & $42(33.6)$ \\
\hline Obscure overt GI blood loss & $34(11.9)$ & $23(14.3)$ & $11(8.8)$ \\
\hline Polyposis syndrome surveillance & $13(4.5)$ & $7(4.3)$ & $6(4.8)$ \\
\hline Celiac disease assessment & $3(1)$ & $3(1.9)$ & $0(0)$ \\
\hline Possible polyp on imaging & $5(1.7)$ & $3(1.9)$ & $2(1.6)$ \\
\hline Other & $17(5.9)$ & $9(5.6)$ & $8(6.4)$ \\
\hline \multicolumn{4}{|l|}{ Mode of ingestion of capsule } \\
\hline Swallowed & $283(99)$ & $160(98.4)$ & $123(98.4)$ \\
\hline Endoscopic transfer from stomach to duodenum & $2(0.7)$ & $1(0.6)$ & $1(0.8)$ \\
\hline Endoscopic placement & $1(0.3)$ & $0(0)$ & $1(0.8)$ \\
\hline \multicolumn{4}{|l|}{ Final position of capsule } \\
\hline Stomach & $6(2.1)$ & $2(1.2)$ & $4(3.2)$ \\
\hline Small bowel & $33(11.5)$ & $19(11.8)$ & $14(11.2)$ \\
\hline Colon & $242(84.6)$ & $137(85.1)$ & $105(84.6)$ \\
\hline Stoma bag & $5(1.7)$ & $3(1.9)$ & $2(1.7)$ \\
\hline Total duration of recording (min) & $507(197-820)$ & $504(197-820)$ & $529(261-614)$ \\
\hline
\end{tabular}

Abbreviations: GI, gastrointestinal; PEG, polyethylene glycol; SBCE, small bowel capsule endoscopy.

Note: Data are given as $n$ (\%) or median (range) except where indicated.

aMean (standard deviation). 
$33(12 \%)$ patients and in the stomach in $6(2 \%)$ patients. No cases of capsule retention occurred.

\section{Small Bowel Capsule Endoscopy Findings and Recommendations}

- Table 2 summarizes the findings and recommendations by indication for patients undergoing SBCE. A total of 144 (50\%) patients had a normal examination. Findings in the remaining examinations included inflammatory changes in $66(23 \%)$ patients, angioectasia in 51 (18\%) patients, polyp(s) in $25(9 \%)$ patients, and a submucosal lesion suspicious for a GI stromal tumor (GIST) in $6(2 \%)$ patients. Strictures were present in nine $(3 \%)$ patients. In six (2\%) patients, a stricture was seen in which the capsule did not traverse during the recording. Both patients had undergone a satisfactory patency capsule assessment in the 3 months prior to the small bowel video capsule study. Capsule retention was excluded in both by a subsequent plain abdominal X-ray.

A recommendation for a further procedure was made in $117(41 \%)$ patients. A recommendation for one or more types of endoscopy was made in $68(24 \%)$ patients. Recommendations were made for a gastroscopy to be undertaken in $9(3 \%)$ patients, a colonoscopy in $17(6 \%)$ patients, anterograde device-assisted enteroscopy in 33 (12\%) patients, and retrograde device-assisted enteroscopy in 12 (4\%) patients. A recommendation for cross-sectional imaging was made in the reports of $14(5 \%)$ studies.

SBCE examinations undertaken in the 125 patients referred for investigation of IDA or obscure overt GI blood loss resulted in a recommendation for a further 103 procedures. In the remaining 183 patients, a further procedure was recommended in only 19 cases.

IDA and obscure GI blood loss accounted for recommendations for a further 103 procedures (in 125 patients) compared with a further 19 procedures in the remaining 183 patients.

The most common therapeutic intervention advised was the application of argon plasma coagulation (APC) for the treatment of angioectasia in $5 \%$ of patients (14 patients). In two patients, the angioectatic lesions were deemed to be within the reach of a gastroscope. Colonic polyps were identified in two patients. Three (1\%) were referred for surgery of whom two were found to have polyps that were deemed to be endoscopically unresectable. One had a submucosal mass with features suspicious for a GIST.

A repeat SBCE was felt advisable by the reporting endoscopist in $10(3 \%)$ procedures.

\section{Diagnostic Yield}

The DY for any abnormality was not significantly different between patients receiving PEG and those who underwent a 12-hour fasting with abnormal findings reported for 66 (53\%) patients in the PEG group and 76 (47\%) patients in the group who underwent a 12-hour fasting $(p=0.348$; - Table 3$)$ When the DY for significant findings only was examined, the DY was not significantly different between the PEG and 12-hour fasting groups, with significant findings in 29 (23\%) patients in the PEG group and 52 (32\%) patients in the group who underwent a 12 -hour fasting $(p=0.090)$.
For patients with IDA, the DY for any abnormality was similar between the two groups (23 [55\%] patients in the PEG group and 26 [53\%] patients in the group who underwent a 12-hour fasting [ $p=0.871]$ ). There was a trend toward an increased DY for significant findings in IDA for patients receiving PEG compared with a 12-hour fasting that just missed statistical significance (13 [31\%] patients in the PEG group and 25 [21\%] patients in the group who underwent a 12-hour fasting [ $p=0.06]$ ).

For the finding of any inflammatory lesion, the DY was similar between the two groups (29 [10\%] patients in the PEG group and 33 [12\%] patients in the group who underwent a 12 -hour fasting $[p=0.582])$. The DY for significant inflammation was also similar between the two groups (25 [9\%] patients in the PEG group and 32 [11\%] patients in the group who underwent a 12-hour fasting [ $p=0.979]$ ).

\section{Gastric and Small Bowel Transit Times}

\section{Capsule transit times are shown in - Table 4 .}

Gastric transit times were unaffected by the use of bowel preparation (20 minutes [range: 1-312 minutes] and 19 minutes [range: 1-239 minutes] in patients receiving PEG and those undergoing a 12-hour fasting, respectively [ $p=0.614]$ ). Gastric transit time was also unaffected by age $(r=0.092$, $p=0.125$ ).

Small bowel transit times were unaffected by the use of bowel preparation (272 [range: 72-654] and 249 [range: 72-654] minutes in patients receiving PEG and those undergoing a 12-hour fasting, respectively $[p=0.373])$. Small bowel transit time was also unaffected by age $(r=0.020$, $p=0.745)$.

\section{Completion Rates}

Completion rates (procedures in which the capsule was seen to have completely traversed the small bowel) were unaffected by age and by the total recording time ( - Table 5 ). The capsule completely traversed the length of the small bowel in in 112 (89.6\%) patients in the PEG group and 141 (87.5\%) patients who underwent a 12-hour fasting. Results of the Chi-square test showed no significant difference in completion rates for patients receiving bowel preparation and those without bowel preparation $\left(\chi^{2(1)}=0.183, p=0.712\right)$.

\section{Discussion}

SBCE has been an important advance in the assessment of the small bowel and plays a major role in the diagnostic workup in patients with suspected small bowel pathology. There remain some uncertainties as how best to optimize the use of SBCE in clinical practice.

Guidelines advocate the use of bowel purgative, but the degree to which this affects visualization and DY is unclear and will occur at the expense of adverse effects from PEG ingestion. ${ }^{7}$ In this analysis, PEG $2 \mathrm{~L}$ and 12-hour fasting had a similar DY across all indications. However, in patients referred for investigation of IDA, there was a trend toward an increased DY for significant findings that just missed statistical significance. 


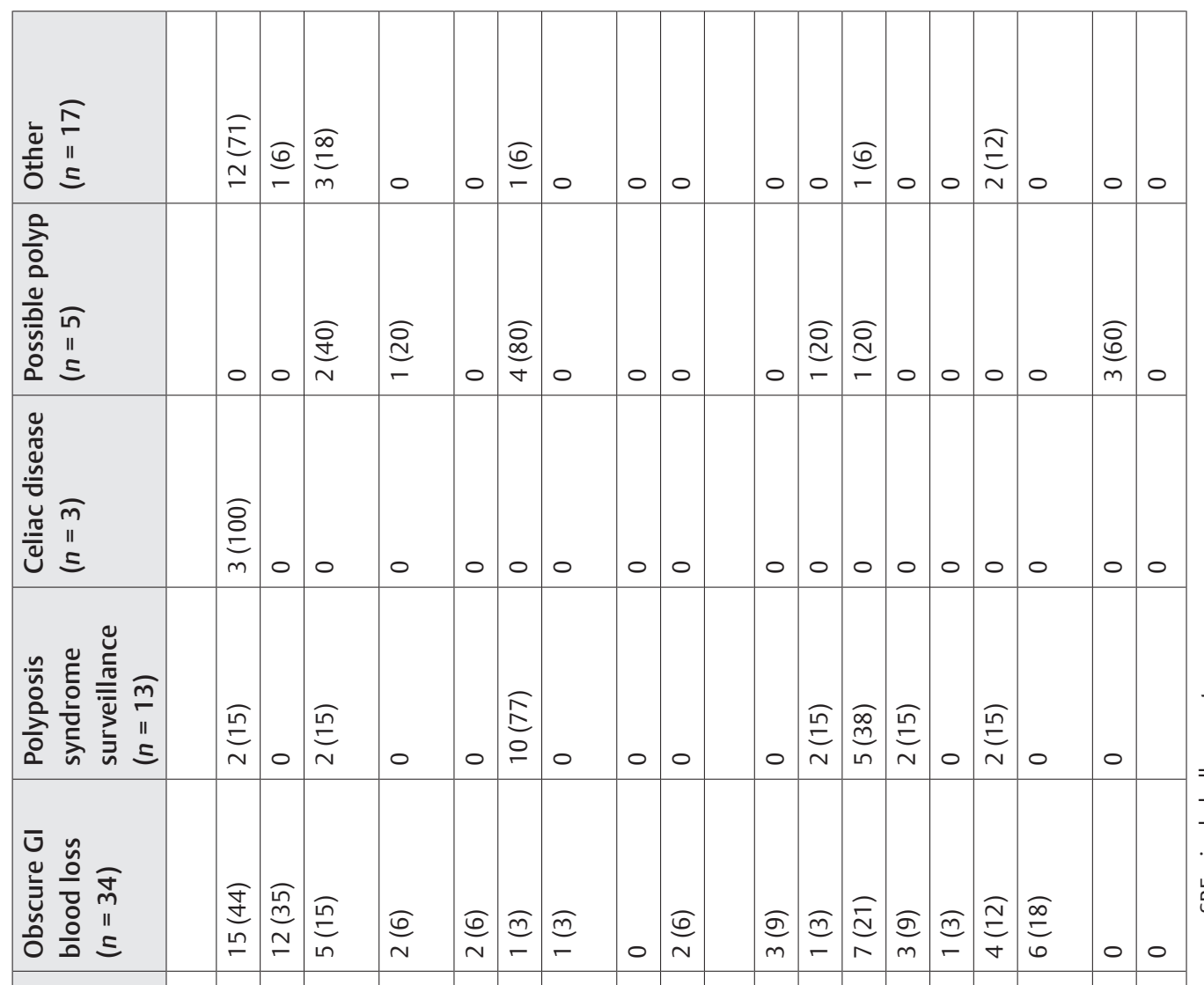

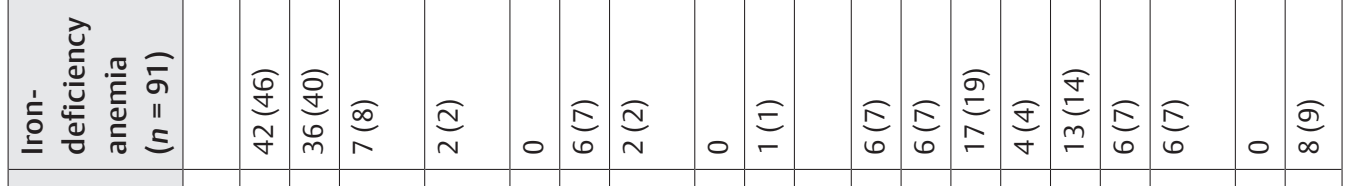

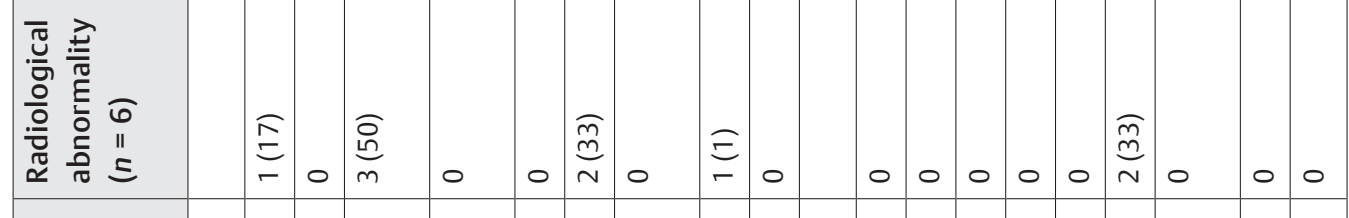

苞

\begin{tabular}{|c|c|c|c|c|c|c|c|c|c|c|c|c|c|c|c|c|c|c|c|c|}
\hline 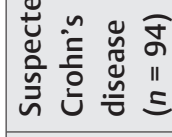 & & $\begin{array}{l}\sqrt{8} \\
0 \\
0 \\
8\end{array}$ & $\underset{\sim}{\sim}$ & $\begin{array}{l}\widetilde{\pi} \\
\stackrel{n}{0} \\
0\end{array}$ & $\underset{-}{\Xi}$ & 0 & $\underset{\ulcorner}{\Xi}$ & $\underset{-}{\Xi}$ & 0 & 0 & & 0 & $\underset{\stackrel{\Omega}{n}}{n}$ & $\underset{\sim}{\mathbb{d}}$ & $\begin{array}{l}\bar{D} \\
N\end{array}$ & 0 & $\begin{array}{c}\hat{O} \\
\stackrel{0}{\sigma} \\
\sigma\end{array}$ & $\underset{\sim}{\widehat{a}}$ & 0 & $\underset{\frac{D}{N}}{d}$ \\
\hline 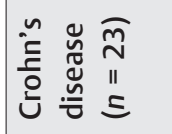 & & $\begin{array}{l}\text { నू } \\
\text { ले } \\
\sigma\end{array}$ & 0 & $\begin{array}{l}\bar{\sigma} \\
\bar{\sigma}\end{array}$ & 0 & 0 & 0 & $\stackrel{\widehat{\rho}}{\sim}$ & $\underset{\sim}{\hat{\sigma}}$ & 0 & & 0 & $\stackrel{\widehat{\sigma}}{\sim}$ & 0 & $\begin{array}{l}\widehat{a} \\
-\end{array}$ & 0 & $\begin{array}{l}\hat{\partial} \\
\stackrel{e}{r} \\
\end{array}$ & 0 & 0 & 0 \\
\hline 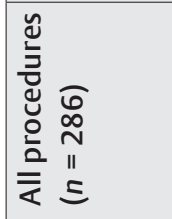 & & 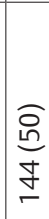 & 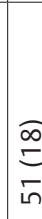 & 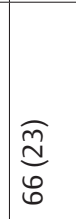 & $\frac{\widehat{\Xi}}{\sigma}$ & $\underset{\sim}{\Xi}$ & $\underset{\stackrel{\sigma}{\sim}}{\stackrel{n}{N}}$ & $\underset{\sigma}{\widehat{\Xi}}$ & $\underset{\mathrm{m}}{\bar{\Xi}}$ & 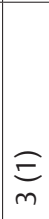 & & $\begin{array}{l}\widehat{\hat{n}} \\
\sigma\end{array}$ & $\begin{array}{l}e \\
\underline{E}\end{array}$ & $m$ & $\begin{array}{l}\mathbb{J} \\
\mathbb{I}\end{array}$ & $\begin{array}{l}\overparen{\Xi} \\
\pm\end{array}$ & 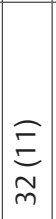 & $\begin{array}{l}\widehat{\mathbb{n}} \\
\pm \\
\square\end{array}$ & $\underbrace{}_{m}$ & 응 \\
\hline & 爰 & $\begin{array}{l}\overline{0} \\
\stackrel{0}{5} \\
\text { Z }\end{array}$ & 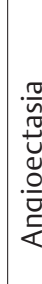 & 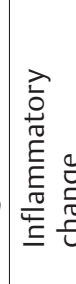 & 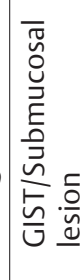 & 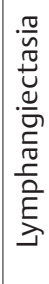 & $\begin{array}{l}\text { ज्ञ } \\
\frac{0}{0} \\
0\end{array}$ & 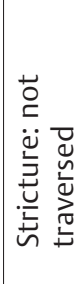 & 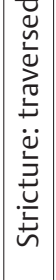 & 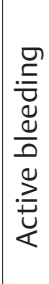 & 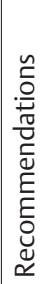 & 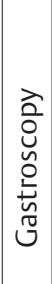 & 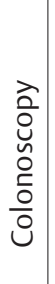 & 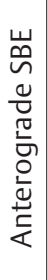 & 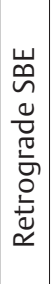 & 这 & 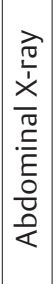 & 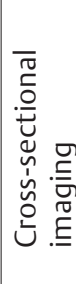 & 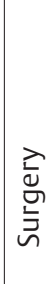 & 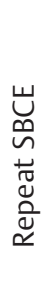 \\
\hline
\end{tabular}


Table 3 Diagnostic yield

\begin{tabular}{|c|c|c|c|c|c|}
\hline \multirow[t]{2}{*}{ Findings } & \multirow[t]{2}{*}{$n$} & \multicolumn{2}{|c|}{$\begin{array}{c}\text { Diagnostic yield } \\
n(\%)\end{array}$} & \multirow[t]{2}{*}{$\chi^{2(1)}$} & \multirow[t]{2}{*}{$p$} \\
\hline & & PEG & 12-hour fasting & & \\
\hline Any abnormality & 286 & $66(53)$ & $76(47)$ & 0.8812 & 0.348 \\
\hline Significant findings & 286 & $29(23)$ & $52(32)$ & 2.869 & 0.090 \\
\hline IDA: any abnormality & 91 & $23(55)$ & $26(53)$ & 0.026 & 0.871 \\
\hline IDA: significant findings & 91 & $13(31)$ & $25(21)$ & 3.745 & 0.06 \\
\hline Any inflammation & 286 & $29(10)$ & $33(12)$ & 0.303 & 0.582 \\
\hline Significant inflammation (LS > 135) & 286 & $25(9)$ & $32(11)$ & 0.001 & 0.979 \\
\hline
\end{tabular}

Abbreviations: IDA, iron-deficiency anemia; LS, Lewis's score; $\chi^{2}$, Chi-square (two-sided significance).

Table 4 Capsule transit times

\begin{tabular}{|l|l|l|l|l|}
\hline \multirow{2}{*}{} & \multicolumn{2}{|c|}{$\begin{array}{l}\text { Transit time (minutes) } \\
\text { Median (range) }\end{array}$} & \multirow{2}{*}{$p$-value } \\
\cline { 2 - 5 } & All examinations & PEG & 12-hour fasting & \\
\hline Ingestion to colon & $282(91-559)$ & $289(91-559)$ & $277(96-506)$ & 0.614 \\
\hline Gastric transit time & $20(1-312)$ & $20(1-312)$ & $19(1-239)$ & 0.922 \\
\hline Small bowel transit time: all examinations & $260(72-654)$ & $272(72-654)$ & $249(72-654)$ & 0.373 \\
\hline $\begin{array}{l}\text { Small bowel transit time: capsule reaches } \\
\text { colon }\end{array}$ & $247(72-654)$ & $255(72-654)$ & $241(74-486)$ & 0.566 \\
\hline
\end{tabular}

Abbreviation: PEG, polyethylene glycol.

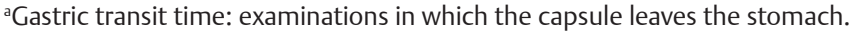

Table 5 Factors affecting completion rate

\begin{tabular}{|l|l|l|l|}
\hline \multirow{2}{*}{} & \multicolumn{2}{|c|}{ Procedural completion } & \multirow{2}{*}{$\boldsymbol{P}$} \\
\cline { 2 - 3 } & Complete & Incomplete & \\
\hline Age $(\mathrm{y})$ & $51.26(1.20)$ & $53.31(2.75)$ & 0.497 \\
\hline Total length of the procedure & $499.00(249-615)$ & $497.50(459-696)$ & 0.806 \\
\hline
\end{tabular}

Note: Complete, capsule left small bowel by end of the recording (final image colon or stoma bag); numbers expressed as median (range) except age (mean \pm standard error of mean).

Studies examining the impact of bowel purgatives on DY have failed to consistently demonstrate a superiority of PEG over a clear liquid (fasting) protocol of preparation. In a meta-analysis of 12 randomized controlled trials, investigating the role of purgatives in SBCE, Gkolfakis et al reported that purgatives did not increase DY or mucosal visualization. ${ }^{3}$ In a meta-analysis of 40 studies, Yung et al reported that although the overall DY was not improved with bowel purgatives (odds ratio $[\mathrm{OR}]=1.11 ; 95 \%$ confidence interval $[\mathrm{CI}]$ : 0.85-1.44), laxatives were associated with an improvement in the quality of small bowel visualization $(\mathrm{OR}=1.60 ; 95 \%$ CI: 1.08-2.06).

Recent studies have indicated that the timing of bowel preparation may be important. Bowel preparation with PEG in a short period before SBCE has been associated with an improvement in small bowel visualization. ${ }^{18,19}$ The question as to whether clear fluids given in a similar timeframe would have a similar efficacy has not been explored. The role of PEG has been challenged by the observation that the image quality at SBCE after bowel preparation with $4 \mathrm{~L}$ of clear fluids is noninferior to $2 \mathrm{~L}$ of PEG and is associated with a significantly reduced rate of side effects. ${ }^{20}$ However, PEG 2 L may be better than clear fluids for visualization of the distal small bowel in particular. $^{12}$

The length of fasting is also an important determinant of small bowel visualization, with a fasting of more than 12 hours resulting in better mucosal visualization than shorter fastings. ${ }^{21}$

The role of bowel cleansing agents in the preparation of patients for SBCE requires further evaluation as any potential benefits will be offset by the high level of adverse symptoms reported by patients using them.

In this retrospective analysis of the performance of SBCE, there was a high yield of positive findings, with abnormalities detected in half of all patients referred for the procedure. Significant findings that prompted a recommendation for a further test or intervention were identified in $41 \%$ of patients. The majority of these procedures ( $84 \%$ ) had been performed to investigate IDA or GI blood loss, despite these being an indication for SBCE in only $44 \%$ of the procedures overall. Also, 
$98 \%$ of culprit lesions identified were outside the reach of a standard gastroscope. This underlines the key role for SBCE in the investigation of IDA and overt GI blood loss. Overall, $16 \%$ of patients were referred for device assisted endoscopy. The impact on other services will need to be considered by centers adopting SBCE into their routine practice.

The main weakness of this study is that data were analyzed retrospectively. Data on the quality of bowel cleansing were not systematically recorded. The only surrogate for an assessment of the quality of mucosal visualization was the number of patients in whom a repeat procedure was recommended. A further SBCE was suggested in nine patients ( $3 \%$ of the total cohort), although the reasons for repeat procedures were not collated.

There is a lack of standardization of reports of DY across most studies of SBCE. The definition of a significant DY adopted by our analysis of SBCE reports is a pragmatic one, based on the need for a further investigation or intervention. However, there is a degree of imprecision inherent in the definition used in this study. As part of a quality improvement initiative, recent guidelines have advocated the use of standardized terminology in the reporting of SBCE and this will promote consistency and uniformity in the description of abnormalities. ${ }^{22}$ While this may not alter the DY of SBCE per se, it will allow a degree of clarity in distinguishing "significant" from "nonsignificant" findings. ${ }^{22}$

\section{Conclusion}

In this retrospective analysis of the performance characteristics of SBCE, SBCE was found to have a high DY with positive findings in half of all patients undergoing the test. Among patients, $16 \%$ required further investigation with device-assisted endoscopy with APC for angioectasia as the most common recommended intervention. Most interventions were required for patients undergoing SBCE for the investigation of IDA or overt GI blood loss, confirming a central role for SBCE in the assessment of these patients.

The use of bowel purgatives in the form of $2 \mathrm{~L}$ of PEG was not superior to a 12-hour fasting on DY or completion rate. PEG $2 \mathrm{~L}$ had no effect on foregut or small bowel capsule transit times. Further work focusing on the quality of bowel cleansing with different bowel preparation regimes that include longer fasting is still needed.

\section{Authors' Contributions}

P.C. conceived the work, supervised collation of the data, analyzed the data, and drafted the manuscript. A.B. and T.S. collated the data. All authors contributed to the revision of the manuscript and approved the final version.

\section{Financial Disclosure}

The authors have not received any payment or services for any aspect of the submitted work that would constitute a conflict of interest..

\section{Conflict of Interest}

The authors have no financial relationships outside the submitted work that would represent a conflict of interest.

\section{References}

1 Bourreille A, Ignjatovic A, Aabakken L, et al; World Organisation of Digestive Endoscopy (OMED) and the European Crohn's and Colitis Organisation (ECCO). Role of small-bowel endoscopy in the management of patients with inflammatory bowel disease: an international OMED-ECCO consensus. Endoscopy 2009;41(7):618-637

2 Pennazio M, Spada C, Eliakim R, et al. Small-bowel capsule endoscopy and device-assisted enteroscopy for diagnosis and treatment of small-bowel disorders: European Society of Gastrointestinal Endoscopy (ESGE) Clinical Guideline. Endoscopy 2015;47(4):352-376

3 Gkolfakis P, Tziatzios G, Dimitriadis GD, Triantafyllou K. Metaanalysis of randomized controlled trials challenging the usefulness of purgative preparation before small-bowel video capsule endoscopy. Endoscopy 2018;50(7):671-683

4 Yung DE, Rondonotti E, Sykes C, Pennazio M, Plevris JN, Koulaouzidis A. Systematic review and meta-analysis: is bowel preparation still necessary in small bowel capsule endoscopy? Expert Rev Gastroenterol Hepatol 2017;11(10):979-993

5 Wu S, Gao Y-J, Ge Z-Z. Optimal use of polyethylene glycol for preparation of small bowel video capsule endoscopy: a network meta-analysis. Curr Med Res Opin 2017;33(6):1149-1154

6 Enns RA, Hookey L, Armstrong D, et al. Clinical practice guidelines for the use of video capsule endoscopy. Gastroenterology 2017;152(3):497-514

7 Rondonotti E, Spada C, Adler S, et al. Small-bowel capsule endoscopy and device-assisted enteroscopy for diagnosis and treatment of small-bowel disorders: European Society of Gastrointestinal Endoscopy (ESGE) Technical Review. Endoscopy 2018;50(4):423-446

8 Niv Y. Efficiency of bowel preparation for capsule endoscopy examination: a meta-analysis. World J Gastroenterol 2008;14(9):1313-1317

9 Rokkas T, Papaxoinis K, Triantafyllou K, Pistiolas D, Ladas SD. Does purgative preparation influence the diagnostic yield of small bowel video capsule endoscopy?: A meta-analysis. Am J Gastroenterol 2009;104(1):219-227

10 Kotwal VS, Attar BM, Gupta S, Agarwal R. Should bowel preparation, antifoaming agents, or prokinetics be used before video capsule endoscopy? A systematic review and meta-analysis. Eur J Gastroenterol Hepatol 2014;26(2):137-145

11 Wi J-H, Moon J-S, Choi M-G, et al; Korea Gut Image Study Group. Bowel preparation for capsule endoscopy: a prospective randomized multicenter study. Gut Liver 2009;3(3):180-185

12 Hookey L, Louw J, Wiepjes M, et al. Lack of benefit of active preparation compared with a clear fluid-only diet in smallbowel visualization for video capsule endoscopy: results of a randomized, blinded, controlled trial. Gastrointest Endosc 2017;85(1):187-193

13 Gralnek IM, Defranchis R, Seidman E, Leighton JA, Legnani P, Lewis BS. Development of a capsule endoscopy scoring index for small bowel mucosal inflammatory change. Aliment Pharmacol Ther 2008;27(2):146-154

14 Cotter J, Dias de Castro F, Magalhães J, Moreira MJ, Rosa B. Validation of the Lewis score for the evaluation of small-bowel Crohn's disease activity. Endoscopy 2015;47(4):330-335

15 Kharazmi AA, Aslani S, Kristiansen MF, Dahl EE, Berner-Hansen M. Indications and diagnostic yield of smallbowel capsule endoscopy in a real-world setting. BMC Gastroenterol 2020;20(1):177

16 Brain J, Schofield J, Gerrish K, et al; Healthcare Quality Improvement Partnership. A guide for clinical audit research and service review: an educational toolkit designed to help staff differentiate between clinical audit, research and service review activities. Available at: https://www.hqip.org.uk/wp-content/uploads/2018/02/ 
hqip-guide-for-clinical-audit-research-and-service-review. pdf. Accessed July 18, 2020

17 Bowalekar SK. Statistics in medical research-II. Measures of central tendency. J Postgrad Med 1993;39(3):166-173

18 Wu S, Zhong L, Zheng P, et al. Low-dose and same day use of polyethylene glycol improves image of video capsule endoscopy: a multi-center randomized clinical trial. J Gastroenterol Hepatol 2020;35(4):634-640

19 Xavier S, Rosa B, Monteiro S, et al. Bowel preparation for small bowel capsule endoscopy - the later, the better! Dig Liver Dis 2019;51(10):1388-1391
20 Placone N, Bahar R, Mann S. The efficacy of 4 liters of clear liquids for small bowel preparation prior to video capsule endoscopy. Clin Endosc 2020(e-pub ahead of print). doi: 10.5946/ ce.2019.213

21 Catalano C, Companioni RAC, Khankhanian P, et al. Video capsule endoscopy: is bowel preparation necessary? J Investig Med 2016;64(6):1114-1117

22 Spada C, McNamara D, Despott EJ, et al. Performance measures for small-bowel endoscopy: a European Society of Gastrointestinal Endoscopy (ESGE) Quality Improvement Initiative. Endoscopy 2019;51(6):574-598 